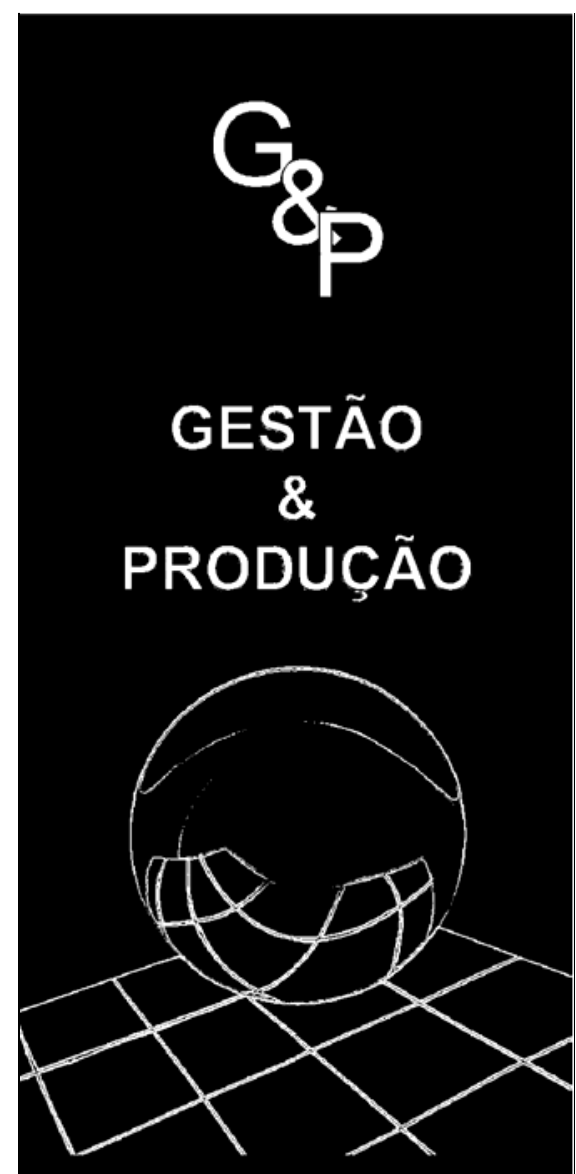

v.5, n.1, p. 60-68, abr. 1998

\section{AGROINDÚSTRIA COOPERATIVA: UM ENSAIO SOBRE CRESCIMENTO E ESTRUTURA DE CAPITAL}

Sigismundo Bialoskorski Neto

Professor Assistente do Departamento de Economia da Faculdade de Economia, Administração e Contabilidade da FEA/USP, Campus Ribeirão Preto Pesquisador do PENSA E-mail: sbialosk@usp.br

Pedro Valentim Marques Professor Associado do Departamento de Economia e Sociologia Rural da ESALQ/USP E-mail: pvmarque@carpa.ciagri.usp.br

\title{
Resumo
}

Este trabalho discute a natureza da empresa agropecuária cooperativa sob o ponto de vista das relações contratuais, isto é, a partir da abordagem da Nova Economia Institucional em particular da Economia dos Custos de Transação. Procura-se analisar o crescimento da empresa e a sua capacidade de adaptação, como condicionantes da sua sobrevivência no mercado, e os problemas advindos do crescimento. Verifica-se que a empresa cooperativa, dado o custo de oportunidade do capital integralizado pelos seus associados e os custos de governança financeira, apresenta maior viabilidade enquanto possui tamanho reduzido. O necessário processo de crescimento induz à empresa cooperativa uma estrutura maior de custos de transação e de agency, quando comparadas com outras formas de organização empresarial. Também é possível indicar a necessidade da mudança na estrutura de capital das cooperativas, por meio de abertura de capital, para que possam sustentar o crescimento e o processo de adaptação, possibilitando a sobrevivência no mercado.

Palavras-chave: economia dos custos de transação, agency, cooperativas, agribusiness.

\section{Introdução}

A s cooperativas são empresas que apresentam uma estrutura particular de organiza- ção, quando comparadas com outras formas empresariais. Na cooperativa não há uma intenção inicial de obtenção de lucros dos negócios, não se recolhe imposto de renda e ao 
final do exercício fala-se em sobras operacionais, que são distribuídas "pro-rata" e proporcionais às operações de cada um dos associados naquele exercício.

Nesta empresa o associado é simultaneamente "cliente" e "proprietário", o que induz a alguns problemas específicos de separação entre a propriedade e o controle, e a alguns problemas de custos associados à necessidade de monitoramento das relações contratuais. Estas características, entre outras, fazem desta empresa um tipo particular de organização que merece uma abordagem específica, principalmente quando se trata dos agronegócios.

A empresa cooperativa é um tipo importante de organização no setor primário da economia. A Organização das Cooperativas Brasileiras (OCB, 1995) indica que existem 1.344 cooperativas agropecuárias respondendo por grande parte da produção de leite, $38 \%$ da soja, e perto de 1/3 do café produzidos no país, entre outros, congregando aproximadamente 1 milhão de associados na área rural.

As 30 maiores cooperativas agropecuárias do país apresentam uma receita operacional líquida total de R\$ 5,9 bilhões (FGV - Agroanalysis, 1996). As cooperativas também participam com perto de $8,47 \%$ das receitas com exportações agrícolas totais e aproximadamente 1,68\% do total das exportações do Brasil, considerando a média de 1991 a 1994. Portanto, são empresas importantes em nossa economia e no universo do chamado agribusiness.

As cooperativas favorecem as relações comerciais entre os seus associados e o mercado, principalmente devido à concentração nestas estruturas, bem como, articulam e coordenam todo um sistema agroindustrial, sob o ponto de vista contratual, de informações e estratégias (BIALOSKORSKI NETO, 1994).

Algumas modificações são sentidas neste setor. Assim, uma cooperativa no SAG da laranja vendeu sua unidade agroindustrial de processamento; uma importante central cooperativa do SAG do leite também se desmobilizou com a venda de sua unidade de processamento.
Uma importante cooperativa do Paraná está reestruturando seu capital de forma a propiciar um controle diferente das suas agroindústrias, inclusive com a participação de capital de não associados. No Canadá e nos EUA também há exemplos de atitudes semelhantes.

Se as cooperativas são empresas importantes no âmbito do agribusiness, inclusive apresentando uma "performance" de negócios apropriada, pode-se perguntar: por que estão ocorrendo estas modificações nas estruturas da empresa cooperativada? Que fatores estão influindo a ponto de fazer com que estas cooperativas busquem novos caminhos?

O presente trabalho tem por finalidade discutir esta situação, bem como identificar de modo inicial quais são os fatores que possivelmente estão determinando este procedimento em firma cooperativada. Neste sentido, são elaboradas algumas contribuições para o tratamento do tema e para possível explicação desta tendência de comportamento estratégico de algumas cooperativas tanto no Brasil, como no exterior.

\section{A Firma e a Economia dos Custos de Transação}

Airma é definida, sob o ponto de vista Aneoclássico, como uma unidade técnica em que se produzem mercadorias de acordo com uma função de produção, na qual os diversos fatores são alocados entre diferentes usos e proporções determinados sempre pelo mecanismo de preços (HENDERSON \& QUANDT, 1988).

COASE (1993) afirma que esta realidade simplificada não se aplica ao mundo real e que a firma pode se organizar e produzir sem estar diretamente influenciada apenas pela sinalização do mecanismo de preços. Há outros fatores a serem considerados, podendo-se indagar: como e por que o mecanismo de preços, da teoria neoclássica, é substituído por outras relações a nível interno da firma? E que relações são estas?

COASE (1993) considerava que havia um custo para o uso do mecanismo de preços, salientando que o custo mais óbvio da organiza- 
ção era o de perceber e descobrir a relevância do próprio mecanismo de preços.

Portanto os custos de negociação e de conclusão de uma determinada transação e/ou troca deveriam ser considerados. Assim, quando estes custos contratuais fossem positivos, ocorreria uma possível cooperação via contratual entre diversos agentes econômicos substituindo o mercado, ou ainda, estas ocorreriam nos limites internos de uma firma como na integração vertical. Determinadas em função destes custos de transação, estas relações contratuais indicariam uma alternativa de organização, que seria mais eficiente que as transações que ocorrem no mercado sinalizadas somente pelo sistema de preços.

A firma então é entendida como um arranjo de diferentes contratos entre diversos agentes econômicos no qual os custos de transação afetariam não só estes arranjos, mas também a alocação de recursos e a forma com que os bens e serviços são produzidos.

Há portanto custos de transação que devem ser considerados e que são diferentes da simples incerteza nas negociações ou mesmo dos custos de informação. WILLIAMSON (1989) define estes custos de transação como os custos ex-ante de elaboração de um instrumento contratual e os custos ex-post desta negociação. Estes custos não estariam sendo considerados nas funções de custo tradicionais da teoria neoclássica e a firma teria outras razões para se organizar de determinado modo além daquelas decorrentes das economias de escala e escopo ou ainda dos ganhos monopolísticos. Isto é, as organizações estariam preocupadas em economizar em seus custos de transação, preocupadas em alcançar a maior eficiência possível.

É claro então que haveria uma coordenação de atividades econômicas de modo alternativo ao mercado, no âmbito das fronteiras de uma determinada organização. As transações via mercado somente ocorreriam quando os custos de transação tendessem a zero. Na presença destes custos, a forma de organizar as transações seria diferente: por meio de uma outra forma de governança.
WILLIAMSON (1989) formulou um modelo em que a especificidade de um ativo seria um dos determinantes dos custos de transação e estes da forma de governança da organização. A especificidade de um ativo é considerada como um grau de especialização de determinado ativo que não permite que este seja, em diferentes graus, utilizado para outro fim se não aquele ao qual é destinado. É necessário distinguir entre especificidade de ativos que induz a transações específicas de um ativo especializado qualquer que é apenas utilizado para determinado fim.

Deste modo, com uma especificidade maior de ativos a organização tende a ter também maior custo de transação, que faz com que esta firma traga ao seu interior certas atividades, ou de uma forma híbrida, procure um relacionamento contratualmente mais estável, que possibilite sempre minimizar os seus custos de transação.

A especificidade dos ativos pode ser de diversas espécies como: de lugar ou geográfica, quando a localização é importante; de caráter humano, quando o capital humano passa a ser específico para a organização; de tempo, quando a dimensão temporal induz a uma situação específica, ou ainda física, quando relacionada a características físicas dos diversos estágios de produção; dedicada, quando envolve investimentos específicos para atender a alguns atores econômicos também específicos.

A formulação destas idéias é importante para o entendimento deste ensaio. De modo simplificado, WILLIAMSON (1989) desenvolve o modelo mostrado na Figura 1, em que se pode observar o comportamento dos custos de transação em função da especificidade de ativos e o resultante modo alternativo com que a firma organiza as suas transações: a governança. No modelo, $\mathbf{M ( k )}$ representa as transações via mercado, $\mathbf{X ( k )}$ via mista e $\mathbf{H}(\mathbf{k})$ via hierárquica ou de modo interno à empresa. Estes modos de relações contratuais também permitem que seja visualizada uma fronteira de eficiência da firma em função da especificidade de seus ativos.

Este modelo assume que quando não existe a especificidade de ativos, isto é $\mathbf{K}=\mathbf{0}$, os custos 


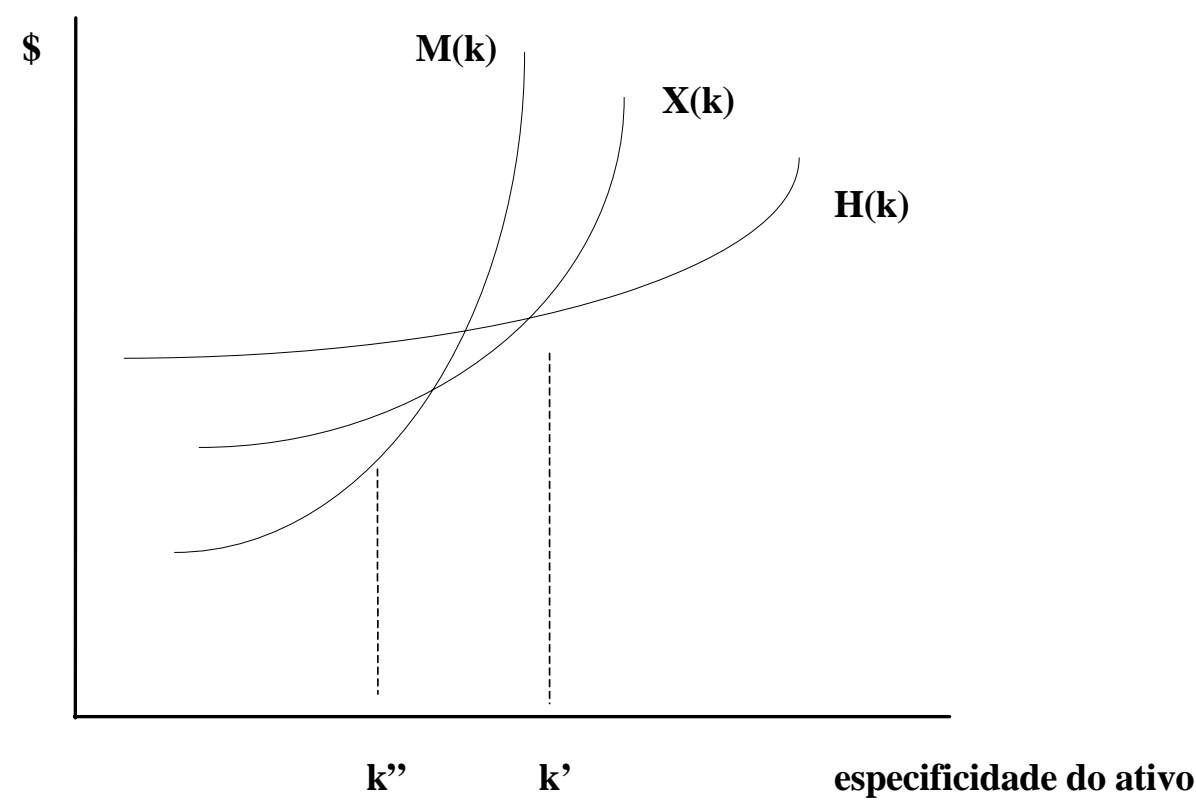

Figura 1 - Governança, ou forma de organização da produção, em função das especificidades de ativos (WILLIAMSON, 1989).

$\mathbf{M}(\mathbf{0})<\mathbf{X}(\mathbf{0})<\mathbf{H}(\mathbf{0})$, ou seja, os custos de transação no mercado são menores do que em outras possibilidades apresentadas.

Também é dado como pressuposto que os custos da forma organizacional via mercado crescem mais que as outras formas quando cresce a especificidade de ativos. Isto é expresso matematicamente como $\mathbf{d}(\mathbf{m}) / \mathbf{d}(\mathbf{k})>\mathbf{d}(\mathbf{x}) / \mathbf{d}(\mathbf{k})>$ $\mathbf{d}(\mathbf{h}) / \mathbf{d}(\mathbf{k})$ e visualizado nas formas convexas das curvas.

ZYLBERSZTAJN (1995) expõe que poderá haver um deslocamento destas três curvas dependendo das modificações nas condições do ambiente de negócios, deslocando o nível de $\mathbf{K}$ para a direita ou para a esquerda fazendo com que estas formas de organização sejam eficientes em diferentes limites possíveis.

A competitividade de determinado SAG também poderá ser analisada de acordo com este instrumental e com o ambiente institucional no qual este opera. Deste modo, a estrutura de governança, ou a forma organizacional, dependerá dos atributos das transações como a incerteza, a frequiência e a especificidade de ativos.

FARINA (1996, p.139) conclui em seu trabalho: "Quanto maior a pressão competitiva (Ambiente competitivo) e quanto menor a tolerância tecnológica (Ambiente tecnológico) tanto maior a importância das estruturas de governança na determinação do desempenho das firmas e dos sistemas. Nesse contexto, o desempenho é sinônimo de competitividade, isto é, da capacidade de sobreviver ou crescer no mercado."

Assim, a questão da adaptação às mudanças do ambiente de negócios é de fundamental importância para a sobrevivência da firma e o sucesso está associado diretamente à forma de governança e coordenação do sistema. Portanto, associado à minimização dos custos de transação.

Nesta análise, há uma questão de adaptação dinâmica ao ambiente de negócios. WILLIAMSON (1989) mostra que mesmo que haja um número grande de transações iniciais, a nível competitivo de mercado, estas poderão 
tender a uma redução, após as inversões duráveis em ativos específicos, objetivando a especialização e as vantagens à frente de rivais competidores no mercado. Esta situação, então, é chamada de "transformação fundamental", isto é, a tendência a uma condição de dependência contratual bilateral, ou de um número muito menor de relações contratuais.

MILGROM \& ROBERTS (1992) apontam que o processo de especialização tem um ponto crucial que é a requisição de uma estrutura mais refinada de coordenação, ou de governança, e que o problema-chave para se alcançar uma efetiva coordenação e adaptação é o fluxo de informações necessárias para determinar a melhor alocação de recursos e estratégias e, portanto, a forma "arquitetural" eficiente para a firma. Como já explicitado, esta última é, sucintamente, um arranjo contratual definido em função dos custos de transação positivos.

Portanto, a questão da adaptação e da coordenação do processo é premissa básica para a eficiência da empresa em uma situação de transformações dinâmicas da realidade de mercado e vai depender diretamente de seu arranjo "arquitetural”.

Quanto às cooperativas, estas são empresas que apresentam toda uma particularidade de relações contratuais e econômicas específicas, de acordo com a Nova Economia Institucional e a Economia Neoclássica, se configurando em uma "arquitetura" empresarial diferente (BIALOSKORSKI, 1995).

Estas formas de organização enfrentam um problema em seu processo de crescimento e especialização, que é a capacidade de adaptação e resposta às novas relações de mercados. Isto ocorre, fundamentalmente, porque estas empresas são formadas como uma coalizão do fator de produção trabalho, necessitando entre outros fatores de aporte de capital e investimentos para inversões em ativos específicos, de modo a possibilitar sua especialização.

Para existir um processo contínuo e eficiente de adaptação e especialização na empresa cooperativista são necessárias algumas condi- ções como o fluxo de informações, a capacidade de coordenação do processo, a alocação eficiente de recursos, de poder, de direitos de propriedade, e a existência de capital para inversões.

Deste modo, havendo cooperação e eficiência da organização, premissas do ensaio, o impedimento ao crescimento e a necessária adaptação das empresas cooperativas seria exclusivo da disponibilidade de capital para as inversões e, logicamente, do seu custo. Dado que é possível se ter um processo de capitalização por meio de empréstimos, ou do mercado financeiro (por meio de ações) e que estes processos diferem entre si, dependentes de como se dá os direitos de propriedade na empresa, a especificidade de ativos, os custos de transação e a especialização da firma, a discussão necessária é de como este processo deve ser encaminhado nas empresas cooperativadas.

\section{A Estrutura de Capital e as Organizações}

$\mathrm{B}$ RICKLEY et alii (1997) consideram a "arquitetura" de uma corporação como função da distribuição dos direitos de decisão a nível interno da firma, dos métodos de pagamentos de resultados e de como se faz o controle do desempenho da empresa. Dependendo de situações específicas a cada uma das firmas, haverá uma combinação diferente na forma como a decisão será tomada, como os resultados serão distribuídos e como será monitorado o desempenho da empresa. A tecnologia, os mercados e o ambiente institucional de regulação (legislação e cultura) são determinantes da estratégia das organizações, bem como da estrutura de seu capital.

DEMSETZ \& LEHN (1985) colocam como determinantes da estrutura de capital de uma empresa a possibilidade da maximização do valor e do tamanho da firma, o potencial de lucro e o controle da organização, e por fim, a regulação. Segundo os autores as mudanças nesta estrutura de capital e de controle das empresas se dariam em função também de custos de transação. 
WILLIAMSON (1989) argumenta que as finanças das corporações podem ser examinadas como projetos de investimentos e distinguidas pela especificidade dos ativos. Assim a estrutura de governança poderia ser entendida também sob o ponto de vista dos instrumentos financeiros e de capitalização das empresas.

Deste modo, o débito decorrente de empréstimos no sistema financeiro, sujeitos às taxas de juros, seria uma estrutura apropriada, isto é, apresentaria menores custos de transação, quando o nível de especificidade de ativos fosse baixo. Quando esta especificidade cresce, aumentam também os riscos das operações financeiras e a estrutura de capital por meio de emissão de ações seria mais apropriada por dividir estes riscos.

Estas formas estariam condicionadas aos custos de transação representados pelos custos ex-ante do estabelecimentos de contratos, ou pelo próprio processo de abertura de capital no mercado, onde se agregariam os custos contratuais relativos a toda estrutura como da Comissão de Valores Mobiliários (CVM), BOVESPA, underwritings para o processo de abertura.

Além desta abordagem da economia dos custos de transação, JENSEN \& MECKLING (1976) alertam para os problemas de agency e controle nestas operações, pois a abertura do capital de uma empresa está diretamente ligado à pulverização do controle empresarial em mãos de um número grande de acionistas. Neste momento, têm-se custos de agency ou seja, o monitoramento de ações entre os "principais" (acionistas) que delegam aos "agentes" (gerentes e/ou gestores da empresa) a delegação de autoridade para agir em seu nome no dia-a-dia do comando da organização.

Nestas condições, JENSEN \& MECKLING (1976) afirmam que ambos são maximizadores de utilidade e, portanto, existem boas razões para se acreditar que o agente nem sempre agirá no melhor interesse do principal e que é geralmente impossível para o principal garantir-se, a custo zero, que o agente tomará decisões ótimas sob o ponto de vista do principal.
Assim, este processo de capitalização dependeria da especificidade de ativos e os custos de transação decorrentes desta, sob o ponto de vista da possível performance futura da empresa quanto aos seus resultados de acordo com o ponto de vista do investidor. Dependeria, também, dos custos associados à divisão entre a propriedade e o controle da organização na ótica do agenciamento.

As cooperativas agropecuárias, devido à sua arquitetura empresarial, determinada institucionalmente pela doutrina e legislação cooperativista, têm certa particularidade quanto aos aspectos apresentados. Inicialmente, a cooperativa pode ser entendida como uma empresa onde uma série de atores econômicos resolvem unir os seus interesses mais imediatos determinados pelas estruturas de mercado concentradas e pela indivisibilidade de alguns fatores de produção na agropecuária. Assim, a primeira leitura pode ser efetuada sob o ponto de vista que o pequeno capital das quotas - parte subscritas inicialmente apresenta um custo de oportunidade muito pequeno, em relação ao mesmo investimento se realizado por um único investidor.

Do ponto de vista neoclássico, considerando (R) o retorno econômico, (t) a quantidade do fator de produção trabalho e (c) a quantidade do fator de produção capital, tem-se uma função do retorno da firma expressa como:

$$
\mathbf{R}=\mathbf{f}(\mathbf{t}, \mathbf{c})
$$

Assim, pela premissa de ganhos tecnológicos dada por $\mathbf{d}(\mathbf{R}) / \mathbf{d}(\mathbf{c})>\mathbf{d}(\mathbf{R}) / \mathbf{d}(\mathbf{t})$, a forma de organização cooperativa só é escolhida quando (t) $>$ (c) .

Inicialmente, como o fator de produção capital é escasso e o fator de produção trabalho é abundante, além de uma intenção inicial de prestação de serviços para os associados e não a maximização do lucro na empresa cooperativa (esta maximização se daria na propriedade particular de cada um dos associados), a escolha de organização seria de uma cooperativa e não uma sociedade anônima. 


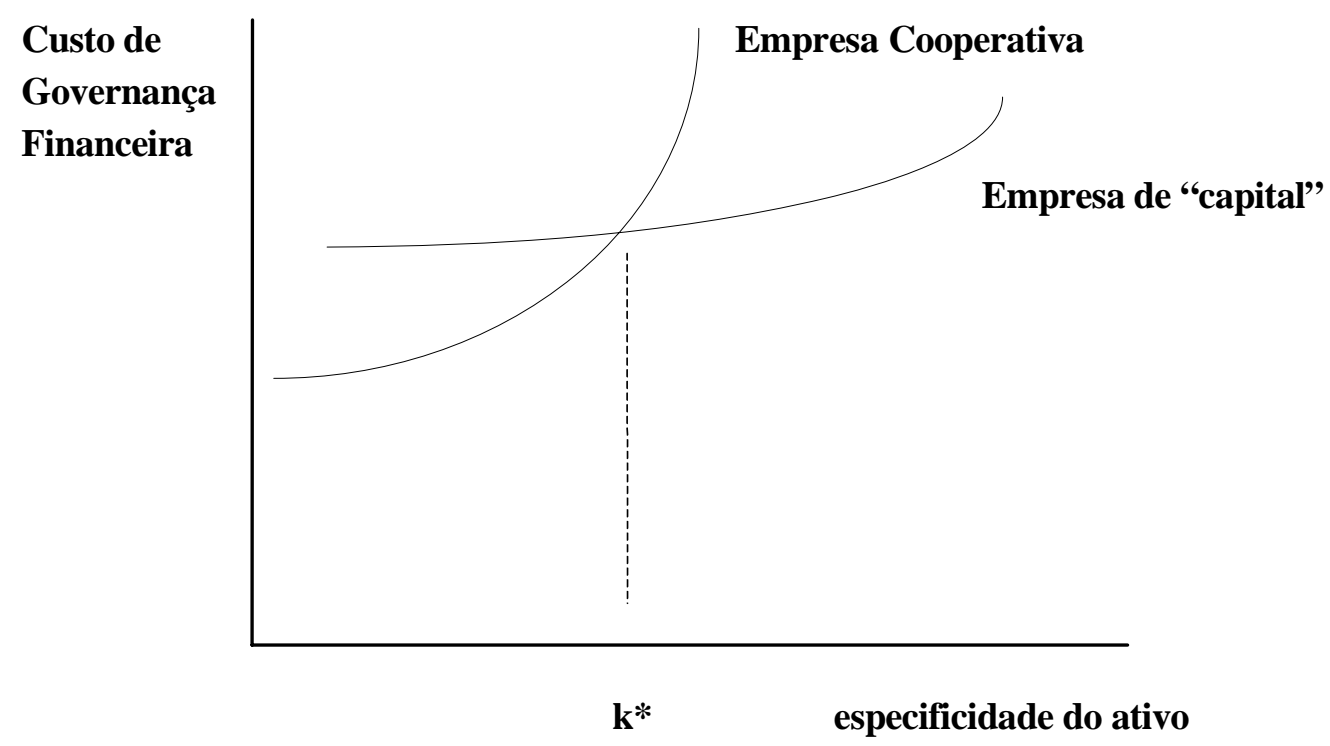

Figura 2 - Custos de governança financeira de empresas cooperativas e não cooperativas em função da especificidade de ativos.

Neste modelo de negócios a empresa cooperativa apresentaria reduzidos custos financeiros, devido ao baixo custo de oportunidade do capital inicial subdividido e aos reduzidos custos de transação devido à reduzida especificidade de ativos.

Dado o início de crescimento e adaptação necessária à melhoria de performance desta empresa e dos seus associados (visando a competitividade), a situação começa a se modificar. Primeiro, ocorre geralmente a especialização de atividades, quando há um crescimento mais do que proporcional da especificidade de ativos, dada a especificidade geográfica, levando ao incremento dos custos de transação, expressos pelas relações contratuais no mercado financeiro, que se refletem também na elevação de juros e estrutura de débito da empresa.

Esta situação seria diferente se a empresa considerada pudesse abrir o seu capital em determinado momento, se capitalizasse de outras formas, ou ainda se crescesse com recursos próprios preservando a sua estrutura de capital. Isto ocorre com as empresas cooperativas que promoveram os seus investimentos prioritaria- mente com fundos próprios, ou empresas que dada a sua complexidade e especificidade de ativos, emitem títulos ou promovem diretamente a abertura de capital com negócios em Bolsa.

Estas afirmações podem ser visualizadas em um gráfico, cujos custos de governança financeira da firma estão em função da especificidade de ativos, mostrados na Figura 2, de acordo com HENDRIKSE (1993). Esta figura mostra que conforme a firma se especializa e cresce, e considerada a sua "arquitetura", ela tem níveis diferentes de custos de governança financeira. Dado um determinado nível de especificidade de ativos, haveria uma destas "arquiteturas" que seria a mais eficiente sob este aspecto particular.

\section{Considerações Finais}

Dode-se considerar, numa primeira aproximaP ção, que possivelmente a razão para que estejam acontecendo algumas desmobilizações nos negócios por parte das cooperativas agropecuárias, e uma clara tentativa de abertura de seu capital, poderia ser explicado pela Nova 
Economia Institucional e sua vertente, a Economia dos Custos de Transação. Empresas cooperativadas, com alta especificidade de ativos, apresentam altos custos de transação e altos custos de agency entre os seus proprietários e gestores, devido à necessária estrutura de participação e aos altos custos de governança financeira de sua capitalização. Por conseguinte, isto faz com que a adaptação aos mercados seja um processo muito custoso.

Nesta possível alternativa de abertura de capital existe uma grande preocupação em manter a identidade da empresa cooperativa, sendo que esta realidade está gerando polêmica e discussão no meio cooperativista internacional. Para compreender esta situação, inicialmente deve-se ater ao fato de que para o sistema cooperativista o aporte de capital e investimentos é de fundamental importância para permitir a constante especialização e competitividade destas empresas no mercado. Para que para que isto ocorra, há diversos caminhos possíveis.

O primeiro caminho é o da capitalização via associados que, apesar de ser o mais fácil e tradicional para o sistema cooperativista, esbarra na dificuldade financeira do setor agropecuário, em particular, do próprio agricultor cooperado que procura a cooperativa exatamente pelo fato desta possibilitar a sua capitalização individual, e não com o intuito de capitalizá-la. O segundo, que foi o caminho mais utilizado ultimamente, é aquele em que a cooperativa busca financiamento via sistema bancário, tomando dinheiro emprestado a um alto custo derivado do nível de juros o que torna este processo muito arriscado frente a possibilidade de altas flutuação de preços das commodities agrícolas.

O terceiro caminho que está sendo de certa maneira percorrido é o da abertura da empresa cooperativista ao capital de risco, no mercado de capitais, possibilitando a atração de recursos a um custo sensivelmente mais baixo, sendo que o investidor também participa dos riscos e resultados do negócio cooperado. Este caminho, hoje em debate, apresenta grandes vantagens, e não só é irreversível para o movimento cooperativista, como também é uma ótima forma para estimular o cooperado a participar nos negócios de sua empresa. Entretanto, deve ser adaptado às características da empresa cooperativista de modo a não representar uma "armadilha” para o sistema.

A abertura de capital com ações preferenciais, sem direito a voto e decisão, encontra um obstáculo quase intransponível nas cooperativas com uma gestão "tradicional". Se a empresa cooperativista não objetiva o lucro, sob o ponto de vista doutrinário, apresentando apenas "sobras de operação", como remunerar preferencialmente ou fazer com que o investidor participe lucrativamente desta empresa? Como ficaria esta aplicação quando comparada a outras aplicações, inclusive em empresas que objetivam exatamente o lucro e a eficiência econômica? Deste modo, inicialmente as cooperativas teriam que objetivar o resultado econômico na sua gestão como condicionante da abertura de capital, de modo competitivo no mercado.

Por outro lado, se as ações permitirem o direito de voto proporcional ao capital investido, isto representa uma nova divisão e alocação nos direitos de decisão na empresa cooperativista. $\mathrm{O}$ princípio democrático de a cada homem um único voto cairia por terra e também parte fundamental da doutrina cooperativista. Pode-se então perguntar: em que uma cooperativa comandada por decisões proporcionais ao capital investido seria diferente de uma sociedade anônima (S/A)? Isto poderia ocorrer mesmo na situação em que estas ações fossem comercializadas somente entre os seus associados, pois a mudança de critério nos processos decisórios permaneceria, podendo se constituir numa "armadilha” para o sistema cooperativista.

Portanto é necessário que este caminho inevitável seja profundamente estudado, à luz das novas teorias, e discutido de modo a representar uma alternativa viável, que una as vantagens da abertura de capital por meio de instrumentos financeiros específicos ou ações com um fortalecimento doutrinário do movimento. 


\section{Referências Bibliográficas}

BIALOSKORSKI NETO, S.: "Agribusiness Cooperativo: A questão do capital nas empresas de trabalho.” Revista Preços Agrícolas, 118(ago), 11-13pp, 1996.

NEVES, E.M. \& MARQUES, P.V.: "Agribusiness cooperativo, eficiência e princípios doutrinários." Revista de Economia e Sociologia Rural, 33(jul-set), 85-121pp, 1995.

"Agribusiness cooperativo: Economia, doutrina, e estratégias de gestão." Piracicaba, ESALQ. Dissertação de Mestrado, 135p, 1994.

BRICKLEY, J.A.; SMITH, C.W. \& ZIMMERMAN, J.L.: Managerial Economics and Organizational Architeture. Chicago, Irwin, 458p, 1997.

COASE, R.H.: "Nobel lecture: The institucional structure of production.” In: WILLIAMSON, O.E. \& WINTER, S.G. The nature of the firm. Origins, evolution, and development. New York, Oxford University Press, 243p, 1993.

DEMSETZ, H. \& LEHN, K.: "The structure of corporate ownership: Causes and consequences." Journal of Political Economy, vol 93, p. 11551177, 1985.
FARINA, M.M.Q.F: "Reflexões sobre desregulamentação e sistemas agroindustriais: A experiência brasileira.” São Paulo, FEA/USP, Tese de Livre Docência, 1996, 156p.

FGV: Agroanalysis, v16 no. 10, 1996.

HENDERSON, J.M. \& QUANDT. R.E.: Teoria Microeconômica - uma abordagem matemática. São Paulo, Pioneira, 417p, 1971.

HENDRIKSE, G.W.J.: "Cooperatives and financial structure.” Tilburg University, 17p. (mimeo.), 1993.

JENSEN, M.C. \& MECKILING, W.H.: "Theory of the firm: Managerial behavior, agency costs and ownership structure." Journal of Financial Economics. vol.3, p.305-360, 1976.

MILGROM, P. \& ROBERTS, J.: Economics, organization and management. New Jersey, Prentice Hall, 619p, 1992

OCB: Anuário do Cooperativismo Brasileiro. 1995.

WILLIAMSON, O.E.: Las instituciones económicas del capitalismo. México, Fondo de Cultura Económica, 431p, 1989.

ZYLBERSZTAJN, D.: "Estruturas de governança e coordenação do agribusiness: Uma aplicação da nova economia das instituições." São Paulo, FEA/USP, Tese de livre docência, 238p, 1995.

\title{
CO-OPERATIVE AGROINDUSTRY: AN ESSAY ON GROWTH AND CAPITAL STRUCTURE
}

\begin{abstract}
This essay discusses the nature of the co-operative agricultural enterprise, from the point of view of contractual relationships, based on the approach of the New Institutional Economics and particularly the Transactions Costs Economics. The intention of this study was to analyze enterprise growth and adaptation capacity, in terms of survival in the marketplace, and the problems due to this growth. It was verified that the co-operative enterprise, given the cost of timing the total capital, and the costs of financial management, shows greater viability while the co-operative is a small enterprise. The necessary growth process imposes greater organizational support for transactions costs and the agency on the co-operative enterprise, when compared with other forms of enterprise organization. It is also possible to indicate the need to change the capital structure co-operatives of the throughout the process of making capital available so the enterprise can sustain the growth and adaptation process, allowing survival in the marketplace.
\end{abstract}

Key words: transactions costs economics, agency, co-operatives, agribusiness. 